\title{
Full Numerical Estimation of Neutrino Mixing Parameters from Solar Neutrino Data
}

\author{
Jai Sam Kim ${ }^{a *}$ and C. W. Kim ${ }^{b}$ \\ ${ }^{a}$ Dept of Physics, Pohang University of Science and Technology, \\ Pohang 790-784, Republic of Korea \\ b School of Physics, Korea Institute for Advanced Study, \\ Seoul 130-012, Republic of Korea
}

(September 17, 1999)

\begin{abstract}
We have numerically computed survival probabilities of solar neutrinos interacting with matter via MSW mechanism in the full three generation formalism with $\Delta m_{23}^{2}=2.2 \times 10^{-3} \mathrm{eV}^{2}$. For $\theta_{13} \lesssim 30^{\circ}$, we confirm the two regions found in the two generation case, (1) with the most likelyhood, small $\sin ^{2}\left(2 \theta_{12}\right) \sim 0.006$, (2) with less likelyhood, large $\sin ^{2}\left(2 \theta_{12}\right) \gtrsim 0.5$. For $\theta_{13} \gtrsim 30^{\circ}$, we find an additional region, (3) with even less likelyhood, $10^{-5}<\Delta m_{12}^{2}<10^{-4} \mathrm{eV}^{2}$ and/or $10^{-4}<$ $\sin ^{2}\left(2 \theta_{12}\right)<0.5$. Assuming that the case (1) is valid, we predict that $\theta_{13} \simeq 15^{\circ}$.
\end{abstract}

PACS numbers: 14.60.Pq; 26.65.+t; 96.60.Jw

Recent Super-Kamiokande experiment [1] shows an evidence for oscillations of the atmospheric neutrinos. The data are in good agreement with two-flavor $\nu_{\mu} \leftrightarrow \nu_{\tau}$ oscillations. These results did establish that neutrinos oscillate and possess non-zero masses. Thus full three-flavor neutrino oscillation analysis is needed for more accurate determination of neutrino masses and mixing anlges. There are many investigations of neutrino oscillations with three neutrino

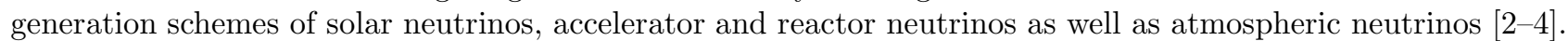

The solar neutrino puzzle is the discrepancy between the standard solar model predictions [5.6] and the results of the solar neutrino experiments. The standard solar model of Bahcall and Pinsonneault predicts that the neutrino event rates are $9.3_{-1.4}^{+1.2} \mathrm{SNU}$ for the chlorine experiment, $137_{-7}^{+8} \mathrm{SNU}$ for the gallium experiment, and $\left(6.62_{-1.12}^{+0.93}\right) \times 10^{6}$ $/ \mathrm{cm}^{2} / \mathrm{sec}$ for the Super-Kamiokande experiment. The observed neutrino event rates are $2.56 \pm 0.16 \pm 0.15$ SNU for Homestake Chlorine experiment [7], $77.5 \pm 6.2_{-4.7}^{+4.3} \mathrm{SNU}$ for GALLEX Gallium experiment [8], 67.2 $2_{-7.0-3.0}^{+7.2+3.5} \mathrm{SNU}$ for SAGE Gallium experiment [9], and $\left(2.44 \pm 0.05_{-0.07}^{+0.09}\right) \times 10^{6} / \mathrm{cm}^{2} / \mathrm{sec}$ for the Kamiokande experiment [10].

Several mechanisms were proposed for the depletion of the solar neutrinos. Among them the Mikheyev-SmirnovWolfenstein (MSW) effect [11,12 has been most popular because it induces large flavor transitions in spite of small vacuum mixing angle in an energy dependent way. Two-flavor MSW solutions 13 14, to the solar neutrino puzzle was fully analysed with high accuracy, including both the day/night effect and spectral information.

In the two-neutrino scheme with the MSW effect it was found that there are two regions in the $\sin ^{2} 2 \theta-\Delta m^{2}$ parameter space that yield two different solutions of the solar neutrino problem: the small mixing angle solution and the large mixing angle solution. The chi-square analysis indicates that the small mixing angle solution is favored [13]. Authors in ref. [15] showed that the high- $\Delta m^{2}$ part of the large mixing angle MSW solution of the solar neutrino problem is disfavored by the Super-Kamiokande atmospheric neutrino data assuming the scheme of neutrino mixing indicated by the result of the reactor neutrino oscillation experiment CHOOZ [16]. This implies that the mixing matrix element $U_{e 3}$ (or $\theta_{13}$ in our parametrization which is the same as that of the Particle Data Group [17]) is small and the oscillations of solar and atmospheric neutrinos are decoupled under the assumption of neutrino mass hierarchy $\Delta m_{12}^{2} \ll \Delta m_{23}^{2},\left(\Delta m_{12}^{2} \equiv m_{2}^{2}-m_{1}^{2}, \Delta m_{23}^{2} \equiv m_{3}^{2}-m_{2}^{2}\right)$.

Since the solar neutrino oscillates into two other flavors, a full analysis may result in some new findings. Especially the angle $\theta_{13}$ may play a non-trivial role. There are many papers in which the MSW effect is considered in threegeneration-neutrino scheme 18 25. However, they used the Landau-Zenor approximate formula for treating flavor conversion in the non-adiabatic resonant region of the solar interior with the assumption that all neutrinos are created at the center of the Sun. Two groups [26,27 implemented numerical methods with two neutrino flavors.

This method of using the Landau-Zenor formula is unreliable when the resonant region and the neutrino production region overlap. In order to demonstrate the situation, we first present a sample of our numerical analysis which shows that for low energy neutrinos the survival probabilities indeed depend very much on their creation sites, $r$. The survival probability of a solar neutrino with energies, $0.3<E_{\nu}<1.0 \mathrm{MeV}$, varies rapidly in the range, $0<r / R_{\odot}<0.2$. Thus $r$-dependence of the survival probability should be taken into account for the $p p$ and Be neutrinos though not as much

*e-mail: jsk@postech.ac.kr 
for the boron neutrinos. That is, one should consider the radial distribution of the solar neutrinos in analysing the gallium experiment data [8,9:28] and the Borexino data [29].

Recently we [30] presented a numerical algorithm for computing the survival probability of an electron neutrino in its flight through the solar core experiencing the MSW effect. We adopted a hybrid method to integrate the neutrino evolution equation in the full three-generation formalism. In order to reduce the number of integrations we used the importance sampling method for sampling the neutrino creation energy and position. In order to reduce the amount of computation in each integration we need to find the optimum radii at which numerical integration is started and stopped. Outside these radii we would use the adiabatic conversion formula. We checked the location of the non-adiabatic resonant regions, where direct numerical integration is performed, by checking if the relative ratios of the off-diagonal and diagonal elements of the Hamiltonian are below a certain limit, say $\gamma_{c}$. Thus the frequency and amount of numerical integrations are greatly reduced. Even with these tactics it is extremely time consuming to solve the neutrino evolution equations for the three-generation case in a large region of the parameter space and it requires deployment of a parallel supercomputer. We developed a parallel algorithm for a message passing parallel computer.

In this paper, we present results of our lengthy numerical computation of the solar neutrino survival probabilities. We will show that the numerical MSW solutions to the solar neutrino problem within the three-generation formalism lead to a new constraint to the neutrino masses and mixing angles. In the three neutrino formalism, we have three vacuum mixing angles $\left(\theta_{12}, \theta_{23}, \theta_{13}\right)$ and one phase to describe mixing between mass and flavor eigenstates. The angle $\theta_{23}$ is irrelevant in the solar neutrino problem and the $\mathrm{CP}$ violating phase may be ignored. Thus adjustable parameters are two mixing angles and two mass squared differences. In conformity with the Super-Kamiokande data on the atmospheric neutrinos, we fixed two parameters, $\Delta m_{23}^{2}=2.2 \times 10^{-3} \mathrm{eV}^{2}$ and $\theta_{23}=43.5^{\circ}$ and searched a wide range of the parameter space spanned by $\Delta m_{12}^{2}, \theta_{12}$, and $\theta_{13}$. We used a $24 \times 21$ logarithmic grid for $\Delta m_{12}^{2}$ and $\sin ^{2}\left(2 \theta_{12}\right)$. We focused on the interesting region, $2 \times 10^{-6} \leq \Delta m_{12}^{2} \leq 4 \times 10^{-4} \mathrm{eV}^{2}$ and $10^{-4} \leq \sin ^{2}\left(2 \theta_{12}\right) \leq 1$.

In our production run, we refined the importance sampling method for sampling neutrino creation energies and radii. For the boron and CNO neutrinos, we chose 64 energy sample points ranging from $0.23 \mathrm{MeV}$ to $18.65 \mathrm{MeV}$ and 60 radius sample points ranging from 0 to $0.353 R_{\odot}$. The three energy values, $0.384,0.862,1.442 \mathrm{MeV}$, are included to evaluate the Be and pep neutrinos more accurately. For the most numerous and model independent $p p$ neutrinos, to be counted by the gallium detectors, we chose 60 energy sample points ranging from $0.23 \mathrm{MeV}$ to $0.4293 \mathrm{MeV}$ and 36 radius sample points ranging from 0 to $0.4 R_{\odot}$. In order to determine the radial limits of the non-adiabatic region, we used a very generous critical value $\gamma_{c}=0.005$, which guaranteed the accuracy of 0.001 for the survival probabilities in the worst case.

We did not include the Earth effect in the calculation. The sensitive parameter region to the Earth density is around $\Delta m_{12}^{2} \sim 10^{-6} \mathrm{eV}^{2}$. In the two-generation scheme the Earth effect does not cause much change of the combined parameter regions for the solar neutrino problems. The data of energy spectrum and day/night variation from the Kamiokande and Super-Kamiokande may give more restriction on the parameter space of neutrino mass and mixing angle. The absence of any day/night variation in the data is consistent with neglecting the MSW resonance effect in the Earth for the electron neutrino. The excluded region by the data of neutrino energy spectrum is around $\Delta m_{12}^{2} \sim 10^{-4} \mathrm{eV}^{2}$ which is outside the combined parameter regions in the two-generation case.

To compute the event rates, we used the $98 \mathrm{SSM}$ flux data taken from [5.6]. We present our results for several values of $\theta_{13}$ in the familiar iso-SNU/FLUX contour format in Figs. 1. In these figures only the mean SNU(FLUX) values are plotted. From these figures we notice three important facts: (1) When $\theta_{13} \lesssim 22.5^{\circ}$, the small mixing angle region where $\sin ^{2}\left(2 \theta_{12}\right) \ll 1$, is the only region where all three curves can come close. In the large mixing angle regions two curves can cross at a time but the crossing points are rather far apart. (2) Beyond $\theta_{13} \gtrsim 22.5^{\circ}$, the gap which the Ga curve passes between the $\mathrm{Cl}$ curve and the Water curve becomes wide and even the small mixing angle solution becomes less likely. (3) When $\theta_{13} \gtrsim 35^{\circ}$, there is no place in the considered region of the $\Delta m_{12}^{2}-\sin ^{2}\left(2 \theta_{12}\right)$ plane where any pair of curves can meet. Thus the likelyhood to have a solution with a large (1-3) mixing angle is very small.

As we can see in the figures the chlorine data are least sensitive to $\theta_{13}$. The Water data are a little more sensitive to $\theta_{13}$ but the shape of the curve does not change. In the small $\Delta m_{12}^{2}$ region with small values of $\theta_{13}$ the Water curves overlap the $\mathrm{Cl}$ curves as both experiments are sensitive mostly to the boron neutrino. Thus it is impossible to determine the mixing angles decisively from these two experiments. However, the gallium detector is sensitive to the $p p$ neutrinos also and its iso-SNU curve crosses the other two curves at large angles and thus most useful for a precision determination of $\theta_{12}$. The figures show that the gallium curves are also very sensitive to a choice of $\theta_{13}$ and they can be used to impose a non-trivial constraint on $\theta_{13}$.

Experimental data contain two kinds of errors, statistical and systematic. We take weighted averages and estimate combined errors as reviewed in [17]. For the $\mathrm{Cl}$ experiment, we use $R_{\mathrm{Cl}}=2.56 \pm 0.23 \mathrm{SNU}$ and for the Water 
experiment, we used $R_{\text {Water }}=(2.44 \pm 0.10) \times 10^{6} / \mathrm{cm}^{2} /$ sec. For the two gallium experiments we take weighted average to get $R_{\mathrm{Ga}}=73.1 \pm 6.6 \mathrm{SNU}$. The flux data from the SSM model contain some theoretical uncertainties. Thus we need to represent the $1 \sigma$ deviation values of SNU(FLUX). Figs. 2 take into account both theoretical uncertainties and experimental errors. The upper curves are from the lower limit of the SSM flux data and the upper values of detection rates of each experiment and vice versa.

For small values of $\theta_{13}$, our result is consistent with the two generation case 13, 14 as expected. Barbieri et. al. [25] performed extensive analysis fo solar neutrino experiments with all three neutrinos. They used the Landau-Zenor approximation formula given in 18,19]. The experimental data are slightly updated since their work. They used, $R_{\mathrm{Cl}}=2.54 \pm 0.20 \mathrm{SNU}$ for $\mathrm{Cl}, R_{\mathrm{Ga}}=75 \pm 7 \mathrm{SNU}$ for $\mathrm{Ga}$, and $R_{\text {Water }}=(2.51 \pm 0.16) \times 10^{6} / \mathrm{cm}^{2} / \mathrm{sec}$ for Water. So direct comparison of our results with their results is not possible. However, we made iso-SNU(FLUX) plots with these data also. For $\theta_{13}=1.5^{\circ}, 15^{\circ}$, our best estimates of $\Delta m_{12}^{2}$ in units of $10^{-6} \mathrm{eV}^{2}$ and $\sin ^{2}\left(2 \theta_{12}\right)$ in units of $10^{-3}$ are $(5.05,5.89)$ and $(6.50,3.99)$ respectively. However, for $\theta_{13}=30^{\circ}$ our estimates are $(7.44,2.20)$, whereas their plots indicate $(\sim 8, \sim 6)$. It seems that their estimates of $\Delta m_{12}^{2}$ are slightly larger than ours but still within the $1 \sigma$ limit. For small values of $\theta_{13}$ we agree with theirs within the $1 \sigma$ limit whereas for large values our estimate of $\sin ^{2}\left(2 \theta_{12}\right)$ is much smaller. In both cases, for large $\theta_{13}$, there is a $1 \sigma$ region where $\Delta m_{12}^{2} \sim 10^{-4} \mathrm{eV}^{2}$.

Due to the theoretical uncertainties and experimental errors, all angular values of $\theta_{13}$ can accomodate all three experiments in broad ranges of $\Delta m_{12}^{2}$ and $\theta_{12}$. For small $\theta_{13}$ the 'small mixing angle' solution gives the most likely solution, with a small patch of 'large mixing angle' region hanging in the upper-right corner. However, for large $\theta_{13}$, the large boomerang shaped region in Figs. 2 allows broad range of values for $\Delta m_{12}^{2}$ and $\sin ^{2}\left(2 \theta_{12}\right)$. Large values of $\Delta m_{12}^{2} \approx 10^{-4} \mathrm{eV}^{2}$ are possible and $2 \cdot 10^{-4}<\sin ^{2}\left(2 \theta_{12}\right)<0.2$ for $\theta_{13}=40^{\circ}$. But its likelyhood seems to be minimal as Figs. 1 indicate. However, the earth effect can broaden the large $\theta_{12}$ region, which we did not consider in this work.

We repeated the computation with $\Delta m_{23}^{2}=5.0 \times 10^{-4} \mathrm{eV}^{2}$, which is the $1 \sigma$ lower limit of the Super-Kamiokande estimates. For small values of $\theta_{13}<22.5^{\circ}$ the contours of mean SNU values (Fig. 1) look almost the same as in the case $\Delta m_{23}^{2}=2.2 \times 10^{-3} \mathrm{eV}^{2}$. However, in the latter case $\left(\Delta m_{23}^{2}=5.0 \times 10^{-4} \mathrm{eV}^{2}\right)$, for large values of $\theta_{13}$ the Water contours are shifted slightly to the left and the Ga contour are pushed further to the upper-left direction, while the $\mathrm{Cl}$ contours are intact. Nevertheless we do not see noticable differences between the two cases. However, with $\Delta m_{23}^{2}=2.0 \times 10^{-4} \mathrm{eV}^{2}$, we noticed non-trivial deviations. In this case (2-3) transitions can take place in addition to the (1-2) transition. Our program has been improved to compute the survival probabilities even for the most general situation when all three transitions occur. We repeated the computation with the same set of $\theta_{13}$. The most significant difference is that the $1 \sigma$ region broadens for large $\theta_{13}$.

We give the values of the small (1-2) mixing angle solutions within $1 \sigma$ range in Table 1 . It is interesting to notice that $\chi_{\min }^{2}$ depends on $\theta_{13}$. Its minimum value occurs when the $\mathrm{Cl}$ and the Water curves overlap perfectly. It can be used to predict the most likely value of $\theta_{13}$ from solar neutrino data alone. In this case the minimum occurs at $\theta_{13}=13.21^{\circ}$.

Table 1. Best estimates of small (1-2) mixing parameters for several values of $\theta_{13}$ obtained by minimizing $\chi^{2}$. Here, we used the definition $\chi^{2}=\sum_{i}\left(\left(R_{i}-R_{i}^{\exp }\right) / \Delta R_{i}^{\exp }\right)^{2}$ with $i=\mathrm{Cl}$, Ga, Water, which is caused purely by the experiments. The $1 \sigma$ bounds are also indicated.

\begin{tabular}{|c|c|c|c|}
\hline$\theta_{13}$ (in deg) & $\Delta_{12} \times 10^{6} \mathrm{eV}^{2}$ & $\sin ^{2}\left(2 \theta_{12}\right) \times 10^{3}$ & $\chi_{\min }^{2}$ \\
\hline 1.5 & $4.81_{-0.80}^{+2.62}$ & $6.29_{-2.69}^{+2.57}$ & 0.04526 \\
5 & $4.89_{-0.78}^{+2.79}$ & $6.10_{-2.76}^{+2.56}$ & 0.05902 \\
10 & $5.52_{-1.42}^{+2.78}$ & $5.22_{-2.44}^{+2.92}$ & 0.07068 \\
12.5 & $6.19_{-2.18}^{+2.15}$ & $4.50_{-3.15}^{+2.12}$ & 0.00237 \\
15 & $6.62_{-2.35}^{+2.44}$ & $4.00_{-1.65}^{+3.17}$ & 0.03779 \\
22.5 & $7.59_{-1.94}^{+2.90}$ & $2.81_{-1.06}^{+1.49}$ & 0.76387 \\
30 & $7.58_{-0.63}^{+8.29}$ & $2.12_{-1.01}^{+1.12}$ & 3.36182 \\
\hline
\end{tabular}

In summary we have computed the solar neutrino survival probabilities $P\left(\nu_{e} \rightarrow \nu_{\beta} ; E_{\nu}, r ; \Delta m_{i j}^{2}, \theta_{i j}\right)$ (1) within the full three generation framework (2) using accurate numerical methods and (3) included the effects due to neutrino creation positions to full extent. We have found that there are three regions in the parameter space where all three solar neutrino experiments can be accomodated within the $1 \sigma$ limit: for small (1-3) mixing angles $\theta_{13} \lesssim 30^{\circ}$, (1) with the most likelyhood, small $\Delta m_{12}^{2} \sim 5 \times 10^{-6} \mathrm{eV}^{2}$ and $\operatorname{small}^{\sin ^{2}}\left(2 \theta_{12}\right) \sim 0.006$, (2) with less likelyhood, large 
$\Delta m_{12}^{2} \sim 10^{-4} \mathrm{eV}^{2}$ and large $\sin ^{2}\left(2 \theta_{12}\right) \gtrsim 0.5$; and for large (1-3) mixing angles $\theta_{13} \gtrsim 30^{\circ},(3)$ with even less likelyhood, $10^{-5}<\Delta m_{12}^{2}<10^{-4} \mathrm{eV}^{2}$ and/or $10^{-4}<\sin ^{2}\left(2 \theta_{12}\right)<0.5$.

From Figs. 1 and Figs. 2 and Table 1, we conclude that the small $\sin ^{2}\left(2 \theta_{12}\right)$ solution with $\theta_{13}$ smaller than $22.5^{\circ}$ will cope with future experiments more likely than the large $\sin ^{2}\left(2 \theta_{12}\right)$ solutions. We estimate the most likely value of $\theta_{13}$ by minimizing $\chi_{\min }^{2}$ with respect to $\theta_{13}$, which comes out to be $\theta_{13}=13.21^{\circ}$ from the Table 1 . Our conclusion is consistent with the $\mathrm{CHOOZ}$ estimate of $\theta_{13}<15^{\circ}$. If the errors in the $\mathrm{Cl}$ and the Water experiments are reduced further and the value of $\theta_{13}$ measured in $\mathrm{CHOOZ}$ and other experiments agree with our prediction, it can serve as an interesting test of the Standard Solar Model and the MSW mechanism.

We would like to thank Dr. J.D. Kim for discussions and Ms. K.S. Lee for making plots and analysing data for the table. We also thank Dr. S.B. Kim for clarifying the Super-Kamiokande data. This work was funded by the POSTECH research fund.

[1] Super-Kamiokande Collaboration, Y. Fukuda et al., Phys. Lett. B 433, 9 (1998); Phys. Lett. B 436, 33 (1998); Phys. Rev. Lett. 81, 1562 (1998).

[2] R.P. Thun and S. McKee, Phys. Lett. B 439, 123 (1998).

[3] V. Barger and K. Whisnant, Phys. Rev. D 59, 093007 (1999).

[4] G.L. Fogli, E. Lisi, A. Marrone, and G. Scioscia, Phys. Rev. D 59, 033001 (1999); G.L. Fogli, E. Lisi, D. Montanino, and G. Scioscia, Phys. Rev. D 55, 4385 (1997).

[5] J.N. Bahcall and M.H. Pinsonneault, Rev. Mod. Phys. 67, 781 (1995).

[6] J.N. Bahcall, S. Basu and M.H. Pinsonneault, Phys. Lett. B 433, 1 (1998).

[7] Homestake Collaboration, B.T. Cleveland et al., Nucl. Phys. (Proc. Suppl.) B 38, 47 (1995); Astrophys. J. 496, 505 (1998).

[8] GALleX Collaboration, P. Anselmann et al., Phys. Lett. B 342, 440 (1995); W. Hampel et al., Phys. Lett. B 388, 384 (1996); Phys. Lett. B 447, 127 (1999).

[9] SAGE Collaboration, A.I. Abazov et al., Phys. Rev. Lett. 67, 3332 (1991); J.N. Abdurashitov et al., Phys. Lett. B 328, 234 (1994); J.N. Abdurashitov et al., astro-ph/9907131.

[10] Super-Kamiokande Collaboration, Y. Fukuda et al., Phys. Rev. Lett. 77, 1683 (1996); Y. Suzuki et al., in Neutrino '98. Y. Fukuda et al., Phys. Rev. Lett. 82, 1810 (1999);

[11] L. Wolfenstein, Phys. Rev. D 17, 2369 (1978).

[12] S.P. Mikheyev and A. Yu. Smirnov, Yad. Fiz. 42, 1441 (1985) [Sov. H. Nucl. Phys. 42, 913 (1985)].

[13] J.N. Bahcall, P.I. Krastev, and A.Yu. Smirnov, Phys. Rev. D 58, 096016 (1998).

[14] N. Hata and P. Langacker, Phys. Rev. D 52, 420 (1995); Phys. Rev. D 56, 6107 (1997).

[15] C. Giunti, C.W. Kim, U.W. Lee, and V.A. Naumov, hep-ph/9902261

[16] M. Apollonio et al., CHOOZ Collaboration, Phys. Lett. B 420, 397 (1998); hep-ex/9907037.

[17] Particle Data Group, C. Caso et al., Eur. Phys. J. C 3, 1 (1998).

[18] S. Parke, Phys. Rev. Lett. 57, 1275 (1986).

[19] T.K. Kuo and J. Pantaleone, Phys. Rev. Lett. 57, 1805 (1986); Phys. Rev. D 35, 3432 (1987); Rev. Mod. Phys. 61, 937 (1989).

[20] C.W. Kim, S. Nussinov, and W.K. Sze, Phys. Lett. B 184, 403 (1987).

[21] A. Baldini and G.F. Giudice, Phys. Lett. B 186, 211 (1987).

[22] S.P. Mikheyev and A. Yu. Smirnov, Phys. Lett. B 200, 560 (1988).

[23] J.N. Bahcall and W.C. Haxton, Phys. Rev. D 40, 931 (1989).

[24] G.L. Fogli, E. Lisi, and D. Montanino, Astropart. Phys. 9, 119 (1998); Phys. Rev. D 54, 2048 (1996).

[25] R. Barbieri et al., hep-ph/9807235, JHEP 9812:017, (1998).

[26] W.C. Haxton and W-M Zhang, Phys. Rev. D 43, 2484 (1991).

[27] G. Fiorentini, M. Lissia, G. Mezzorani, M. Moretti, and D. Vignaud, Phys. Rev. D 49, 6298 (1994).

[28] E. Bellotti et. al., GNO-Proposal, Gran Sasso Lab. (LNGS) INFN/AE-96/27; details at URL site: http://kosmopc.mpi-hd.mpg.de/gallex.html.

[29] Borexino Collaboration, G. Bellini et. al. in TAUP '95, Nucl. Phys. (Proc. Suppl.) B48, 547 (1996); details at URL site: http://almime.mi.infn.it

[30] J.S. Kim, Y.S. Chae and J.D. Kim, Comp. Phys. Comm. 120, 41 (1999). 

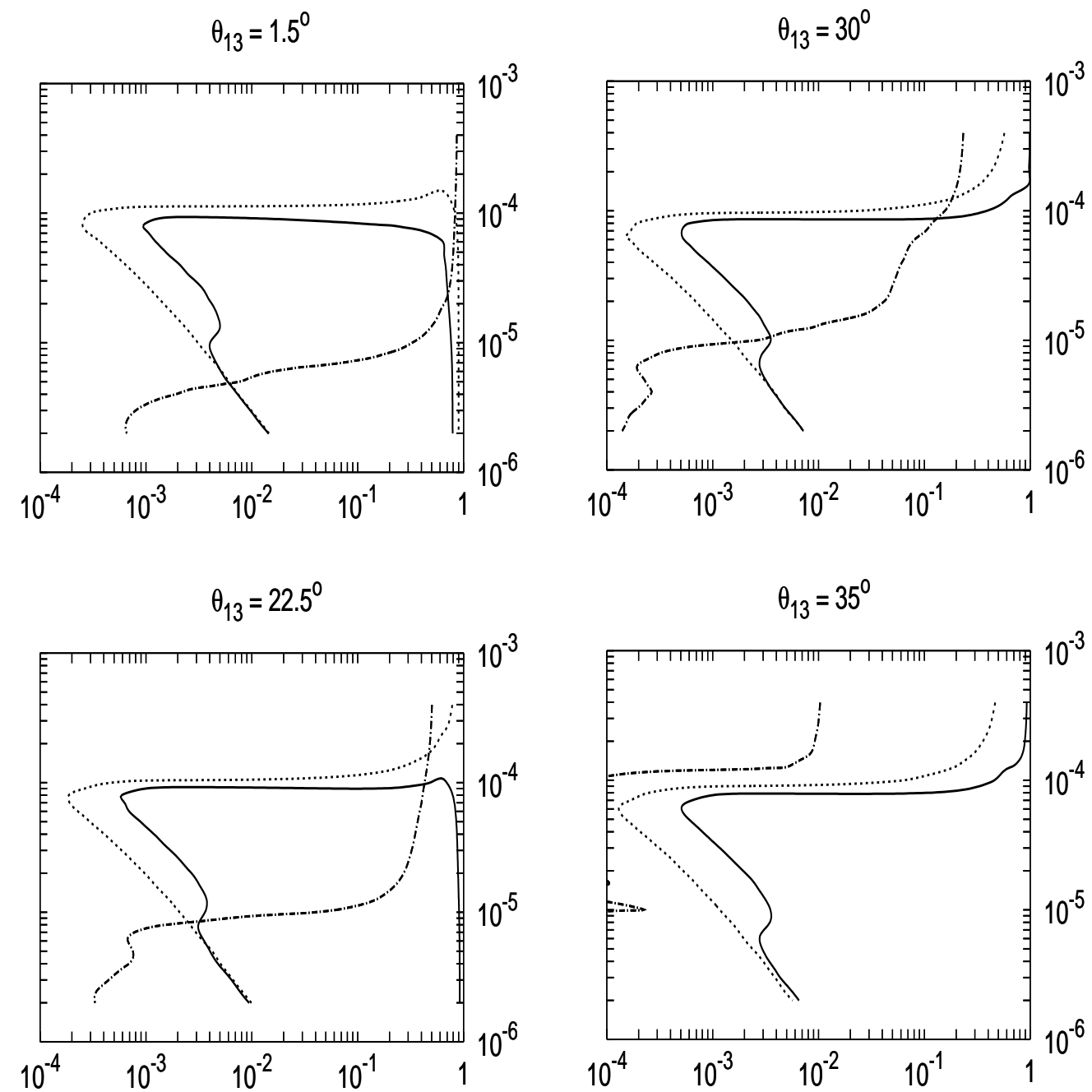

Fig. 1. iso-SNU(FLUX) plots for solar neutrinos with the $\mathrm{Cl}$, Ga, and Water detectors.

Contour values are 2.56 SNU $(\mathrm{Cl}), 73.1 \mathrm{SNU}(\mathrm{Ga})$ and $2.44 \times 10^{6} / \mathrm{cm}^{2} / \mathrm{sec}$ (Water) and they are represented by solid, dash-dot, and dotted curves respectively. In each plot the vertical axis refers to $\Delta m_{12}^{2}$ in $\mathrm{eV}^{2}$ and the horizontal axis to $\sin ^{2}\left(2 \theta_{12}\right)$. We used $\Delta m_{23}^{2}=2.2 \times 10^{-3} \mathrm{eV}^{2}$. 
This figure "JSK_F2.gif" is available in "gif" format from: http://arxiv.org/ps/hep-ph/9909428v1 